\title{
Gliadin uptake in human enterocytes. Differences between coeliac patients in remission and control individuals
}

\author{
S Friis, E Dabelsteen, H Sjöström, O Norén, S Jarnum
}

\begin{abstract}
The pepsin trypsin digest of the wheat prolamin gliadin (PT-gliadin) is deleterious to the small intestinal mucosa of coeliac patients. The handling of PT-gliadin by the intestinal epithelium in coeliac patients in remission and control individuals was investigated by in vivo instillation of PT-gliadin. The uptake of PTgliadin was monitored by immunofluorescence microscopy of intestinal biopsy specimens, using affinity purified PT-gliadin antibodies. Control individuals show weak staining in the apical region of the enterocytes thereby showing an uptake of PT-gliadin. Coeliac patients have a conspicuous fluorescence in relation to the lateral membrane/intercellular space of enterocytes and intense staining intracellularly in the apical region. There is only weak staining in the enterocytes after the instillation was terminated, indicating an intracellular clearance. The study shows that normal enterocytes are able to take up PT-gliadin. The increased uptake in coeliac patients might be of importance for the pathogenesis either by direct toxicity or by presentation to immunocompetent cells. Furthermore, the results are in agreement with the suggestion of a functional alteration in the zonula occludens in the intestinal epithelium of coeliac patients. (Gut 1992; 33: 1487-1492)
\end{abstract}

Department of Medicine A, Division of Gastroenterology, Rigshospitalet, Copenhagen, Denmark S Friis S Jarnum

Department of Biochemistry C, The Panum Institute, University of Copenhagen, Copenhagen, Denmark

H Siöström O Norén

Department of Oral Diagnosis, Royal Dental College, Copenhagen, Denmark

E Dabelsteen

Correspondence to: Steffen U Friis, Department of Medicine A, Division of Gastroenterology A2151, Rigshospitalet, Blegdamsvej 9 , DK-2100 Copenhagen $\varnothing$ Denmark.

Accepted for publication 4 June 1992
Coeliac disease is characterised by flattened jejunal mucosa which causes malabsorption. It is well known, that in susceptible individuals, the intestinal mucosal damage is induced by polypeptides in the prolamin fraction of wheat, barley, rye and possibly oats. Complete restoration occurs after a period of prolamin free diet.

Frazer et al ${ }^{1}$ showed in 1959 that a pepsin trypsin digest of the wheat prolamin gliadin (PT gliadin), was deleterious when ingested orally by coeliac patients. The authors used a rise in faecal fat as an objective measure for toxicity. Bramble et $a l^{2}$ supplemented this observation by administering a PT-gliadin preparation directly into the proximal jejunum of adult coeliac patients in remission and controls. The changes in brush border enzymes and morphology were conspicuous within four hours, thereby showing that the mucosal damage, characteristic for coeliac disease, occurred rapidly. The exact toxic component(s) of gliadin has not been identified and the pathogenetic mechanism of coeliac disease remains uncertain.

It is thus well documented that both gliadin and PT-gliadin are toxic to coeliac patients when injested orally. The intraluminal processing of ingested gliadin has only been partly studied, and a difference in the handling of gliadin in coeliac patients and healthy controls has not been demonstrated. It must be presumed, however, that fragments of gliadin are present in the chyme of coeliac patients. Several workers have investigated the breakdown of gliadin at the level of the enterocytes, using homogenates of intestinal biopsies from patients and controls. ${ }^{3+}$ Consistent quantitative differences in the enzymatic activities have not been definitely demonstrated, even though Cornell et $a l^{5}$ did show, that a certain fraction (no 9) of a gliadin digest was less well hydrolysed by enzymes from mucosal biopsies of coeliac disease patients in remission. The capability of a breakdown of gliadin peptides by the intestinal brush border enzymes alone have been studied in detail by Bruce et al. ${ }^{6}$ It was shown that both brush border from coeliac patients and controls were able to hydrolyse gliadin completely in vitro.

As there is no apparent difference in the digestion of gliadin in coeliac patients and control individuals, there may be a difference in the intestinal uptake and/or intracellular processing of the partly digested gliadin. Gliadin fragments present at the microvillar membrane may be taken up by apical endocytosis. The internalised fragments may subsequently be processed intracellularly and/or be directly toxic in coeliac patients. Optionally, fragments could be transcytosed across the enterocyte and presented at the basolateral membrane for immunocompetent cells, maybe initiating a pathological response in coeliac patients. A pathological route could alternatively be by an intercellular uptake, passing defect tight junctions. In this case, the gliadin fragments may be directly toxic through a binding/uptake via the basolateral membrane. However an in vivo uptake of polypeptides by human enterocytes has so far not been demonstrated.

The purpose of the current investigation was to show a possible binding or uptake of PT-gliadin in the enterocytes, and to compare the handling of the components between coeliac patients and controls. The study was carried out by in vivo instillation of PT-gliadin in the jejunum of two control individuals and three coeliac patients in remission. The fate of the PT-gliadin was monitored by immunofluorescence microscopy of intestinal biopsy specimens obtained before, during, and after the instillation, using an affinity purified antibody against PT-gliadin. 
Methods

MATERIALS

Gliadin, pepsin (P 7012), and trypsin (T 8642) were purchased from Sigma Chemical Co, St Louis, MO, USA, and Coomassie Brilliant Blue R-250 was a product of Merck, Darmstadt, Germany. Triton X-100 was from Serva Feinbiochemica $\mathrm{GmbH}$ and Co, Heidelberg, Germany, while the bovine serum albumin was from Fluka Chemie, Buchs, Switzerland. Statens Seruminstitut, Copenhagen, Denmark provided Freund's incomplete adjuvant and aprotinin was a product of Novo-Nordisk A/S, Bagsværd, Denmark. The CNBr-activated Sepharose 4B and protein-A Sepharose CL-4B were from Pharmacia Fine Chemicals, Uppsala, Sweden. Dakopatts, Glostrup, Denmark, provided the fluorescein isothiocyanate conjugated goat antirabbit IgG antibody. The hydraulic suction tube for multiple biopsies was obtained from Quinton, Seattle, Wash, USA. The airfuge tubes $(0.2 \mathrm{ml})$ were a product of Beckman, Palo Alto, Calif, USA, and the microassay for measurement of protein was from Bio Rad, Richmond, Calif, USA. All other chemicals were of analytical grade. The interference filter was from Optisk Laboratorium, Lundtofte, Denmark.

\section{ENZYMATIC DIGESTION OF GLIADIN}

The enzymatic digestion of gliadin was carried out essentially as described by Bronstein et al. ${ }^{7}$ One gram of gliadin was dissolved in $10.0 \mathrm{ml} 0.1$ $\mathrm{mol} / \mathrm{l} \mathrm{HCl}, 20 \mathrm{mg}$ pepsin was added and after digestion (two hours, $37^{\circ} \mathrm{C}$ ) $\mathrm{pH}$ was adjusted to 8.0 by $5.0 \mathrm{~mol} / \mathrm{l} \mathrm{NaOH}$. The gliadin was then further digested with $20 \mathrm{mg}$ trypsin (four hours, $37^{\circ} \mathrm{C}$ ) at constant stirring. Inactivation of trypsin was achieved by heating $\left(90^{\circ} \mathrm{C}\right.$, three minutes). Insoluble material was removed by centrifugation $(10000 \times g, 30$ minutes). The molecular weight of the polypeptides in the PT-gliadin was estimated by sodium dodecyl sulphate polyacrylamide gel electrophoresis followed by staining with Coomassie Brilliant Blue R-250 as earlier described in detail. ${ }^{8}$ The PT-gliadin was prepared within 24 hours before use and kept at room temperature.

\section{PT-GLIADIN-SEPHAROSE 4B}

PT-gliadin was dialysed for 16 hours at $4^{\circ} \mathrm{C}$ against the coupling buffer $(0.1 \mathrm{~mol} / 1$ sodium hydrogencarbonate, $\mathrm{pH} 8 \cdot 3,0.5 \mathrm{~mol} / 1 \mathrm{NaCl})$. A total amount of $15 \mathrm{mg}$ protein was coupled to $0.6 \mathrm{~g}$ (dry weight) $\mathrm{CNBr}$-activated Sepharose $4 \mathrm{~B}$ according to the instructions of the manufacturer. The coupled Sepharose was packed in a $1.5 \mathrm{~cm} \times 1.3 \mathrm{~cm}$ column and carefully washed with a $50 \mathrm{mmol} / \mathrm{l} \mathrm{Tris} / \mathrm{HCl}, \mathrm{pH} 8 \cdot 0,0 \cdot 15 \mathrm{~mol} / \mathrm{l}$ $\mathrm{NaCl}$ buffer.

\section{HUMAN ENTEROCYTE PROTEIN-SEPHAROSE 4B}

$A$ biopsy specimen was taken under radiographic control from the jejunum of a healthy individual at the ligament of Treitz. The biopsy was instantly frozen and kept at $-80^{\circ} \mathrm{C}$ until further use. The specimen was then thawed at room temperature and homogenised for three minutes on ice in 4 volumes of $50 \mathrm{mmol} / 1 \mathrm{Tris} / \mathrm{HCl}, \mathrm{pH}$ 8.0 containing $1 \%$ Triton $\mathrm{X}-100$ and $2.8 \mu \mathrm{g}$ aprotinin $/ \mathrm{ml}$. The homogenisation was performed in a $0.2 \mathrm{ml}$ airfuge tube, using a Teflon pestle, and the homogenate was subsequently centrifuged for one hour at $50000 \times g$. The protein concentration in the supernatant was determined by use of a microassay version of the Coomassie Brilliant Blue G-250 method, ' using bovine serum albumin as a standard. A total amount of $15 \mathrm{mg}$ enterocyte protein was coupled to 0-6 g (dry weight) CNBr-activatd Sepharose $4 \mathrm{~B}$ as described above.

\section{RABBIT ANTIBODIES AGAINST PT-GLIADIN}

\section{Immunisation}

A $1 \mathrm{mg} / \mathrm{ml} \mathrm{PT-gliadin} \mathrm{solution} \mathrm{was} \mathrm{prepared} \mathrm{by}$ dilution of the gliadin digest with distilled $\mathrm{H}_{2} \mathrm{O}$ and mixed with an equal amount of Freund's incomplete adjuvant. A rabbit was injected intracutaneously with $400 \mu \mathrm{l}$ of the solution. After six weeks the rabbit was bled for $10 \mathrm{ml}$. The immunisation was carried out every four weeks and $10 \mathrm{ml}$ of blood was collected one week after each injection and analysed for reactivity against PT-gliadin by dot blotting. ${ }^{10}$ After 42 weeks the rabbit started to produce antibodies against PTgliadin.

\section{Purification}

The IgG fraction was isolated from $15 \mathrm{ml}$ rabbit serum on a protein-A Sepharose CL-4B. ${ }^{11}$ The PT-gliadin reactive fraction was affinity-purified by using the PT-gliadin-Sepharose 4B column according to the procedure earlier described in detail. ${ }^{12}$ In order to eliminate possible antibodies cross reacting with proteins originating from human enterocytes, the purified PT-gliadin immunoglobulin fraction was passed through the human enterocyte protein Sepharose 4B column. The fractions were analysed for reactivity against PT-gliadin and solubilised proteins from human enterocytes by dot blotting. ${ }^{10}$ The fractions containing reactivity against PTgliadin only were pooled and stored at $4^{\circ} \mathrm{C}$ after the addition of sodium azide. The final IgG concentration $(0 \cdot 14 \mathrm{mg} / \mathrm{ml})$ was determined spectrophotometrically from the absorbance at $280 \mathrm{~mm}$.

INSTILLATION OF PT-GLIADIN

Two control individuals and three adult coeliac patients in remission agreed to take part in the study. No significant intestinal pathology or malabsorption was found in the controls. The coeliac patients were originally diagnosed according to the procedure recommended by the European Society for Paediatric Gastroenterology and Nutrition ${ }^{13}$ and had all been on a complete gluten free diet for at least five years and did not present symptoms indicative of active disease. All those included in the study presented IgG and IgA gliadin-antibody titres within normal range. ${ }^{14}$

The Quinton multiple biopsy instrument was 
Figure 1: Gliadin was enzymatically digested with pepsin and trypsin (PTgliadin). The molecular weight of the polypeptides in the digest was estimated by SDS-polyacrylamide gel electrophoresis followed by staining with Coomassie

Brilliant Blue R-250.

(A) $15 \mu \mathrm{g}$ of undigested gliadin. (B) $45 \mu \mathrm{g} P T$ gliadin. A rabbit was immunised with PT-gliadin. The IgG fraction of a serum sample was isolated and the antibodies reacting with $P T$ gliadin was purified by affinity chromatography. A dot blotting experiment $(C)$ demonstrated, that the purified antibodies did not cross-react with endogenous antigens in the human enterocytes. (1) $5 \mu \mathrm{g} P T$ gliadin. (2) $5 \mu \mathrm{g}$ gliadin. (3) $5 \mu \mathrm{g}$ proteins from $a$ homogenate of a jejunal biopsy specimen from a healthy individual.

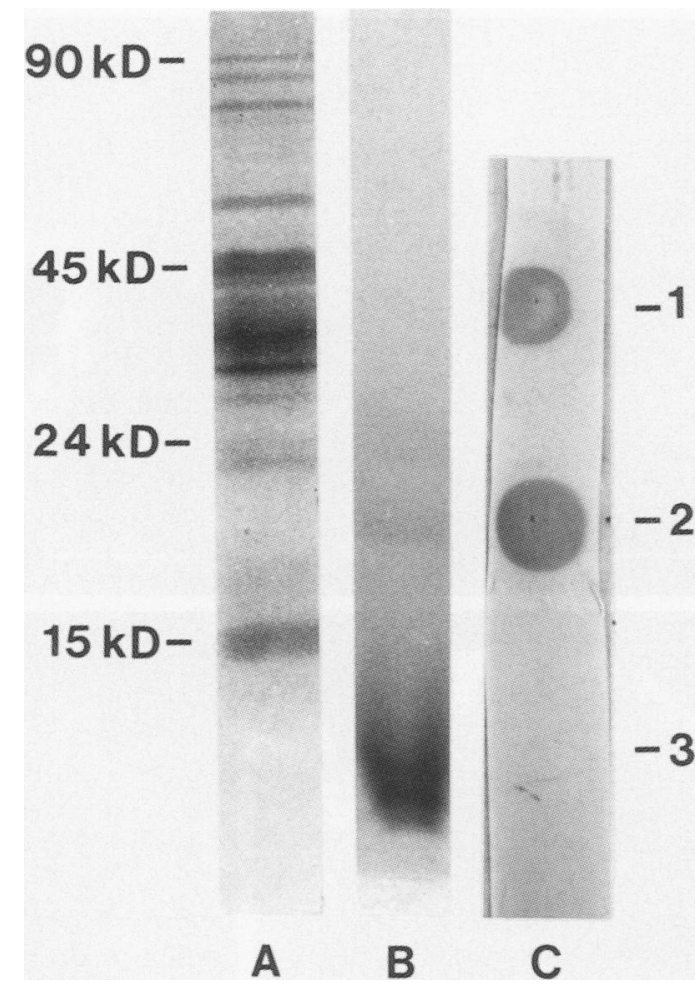

introduced to a position corresponding to the ligament of Treitz and left in place during the study. Before the instillation of PT-gliadin, a biopsy specimen was taken and studied under a stereomicroscope in order to ensure a normal jejunal villous structure. These biopsies were later investigated in a light microscope to confirm a normal mucosa. Each person was challenged with $50 \mathrm{ml} \mathrm{PT}$-gliadin $(8 \mathrm{mg} / \mathrm{ml})$ over a period of 40 minutes through the biopsy tube. The position of the tube was repeatedly controlled by radiography. Biopsies were taken after 20 , 40 , and 75 minutes from the control persons and after 20, 40, and 60 minutes from the coeliac disease patients. Each biopsy specimen was sectioned into four pieces and immediately frozen in a drop of methylbutane in liquid nitrogen and kept at $-80^{\circ} \mathrm{C}$ for subsequent studies.

\section{IMMUNOFLUORESCENCE MICROSCOPY}

Sections $(5 \mu \mathrm{m})$ were cut from the frozen biopsies on a cryostat microtome. They were incubated for one hour at $4^{\circ} \mathrm{C}$, with a series of PT-gliadin IgG rabbit antibodies $(0.07 \mathrm{mg} / \mathrm{ml}$, $0.028 \mathrm{mg} / \mathrm{ml}, 0.014 \mathrm{mg} / \mathrm{ml}$ ) diluted with phosphate buffered saline, $\mathrm{pH} 7 \cdot 4$. The sections were washed three times for five minutes in the phosphate buffered saline and goat antirabbit IgG conjugated to fluorescein isothiocyanate was applied (one hour at $4^{\circ} \mathrm{C}$ ). The washing procedure was repeated and the sections mounted in buffered glycerol containing $\alpha$-phenylenediamine to prevent fading of the fluorescence. ${ }^{15} \mathrm{~A}$ Zeiss epifluorescence microscope equipped with interference filters for fluorescein isothiocyanate was used for the examination. The control experiments included both use of a non-immune serum as the first layer and staining with conjugate alone. Additionally, a solution of the puri- fied primary antibody absorbed with PT-gliadin was tested.

The sections were evaluated blindly twice with two months interval. The exposure of control experiments was approximately doubled in order to achieve a reasonable background structure.

ETHICS

The study was approved by the local ethical committee.

\section{Results}

The pepsin trypsin digest of gliadin was water soluble and contained polypeptides with maximal molecular weight of approximately $20 \mathrm{kD}$ as estimated by sodium dodecyl sulphate/ polyacrylamide gel electrophoresis (Fig 1B). The dot blotting experiments showed that the immunoadsorbent purified PT-gliadin IgG antibodies did not cross-react with endogenous antigens from human enterocytes (Fig 1C).

Fluorescence was not detected in control experiments, which included use of a nonimmune serum as the first layer or incubation with fluorescein isothiocyanate conjugate alone. Furthermore, staining could not be observed when the solution of purified primary antibody absorbed with PT-gliadin was used as the first layer (figure not shown).

UPTAKE OF PT-GLIADIN IN CONTROL INDIVIDUALS PT-gliadin could not be detected before the instillation in normal individuals (figure not shown). During the instillation reactivity was detected extracellulary at the luminal surface. In the sections of intestinal biopsies obtained after 20 (Fig 2B) and 40 minutes a faint colouring was seen apically in the enterocytes. The staining was homogeneous and without an apparent localisation in granules. There was no difference in the staining between the villous and the crypt regions. Staining could not be demonstrated in relation to the basolateral membrane/intercellular space. After 75 minutes (35 minutes after the instillation was terminated) only very weak intracellular colouring was seen in the two control individuals. Furthermore, the intracellular staining was only observed when the most concentrated immunoglobulin solution $(0.07 \mathrm{mg} /$ $\mathrm{ml}$ ) was used. The sections incubated with the more diluted antibodies displayed only extracellular staining at the luminal surface of the enterocytes.

\section{UPTAKE OF PT-GLIADIN IN COELIAC PATIENTS}

The results of the immunofluorescence studies of sections from the intestinal biopsy specimens from coeliac patients are shown in Fig 2A, C-F. There was no staining for PT-gliadin in relation to the enterocytes before the instillation (Fig 2A). Staining was seen in goblet cells in one of the coeliac patients. This observation occurring in only one patient is thus considered of no importance for the study.

After 20 minutes of instillation a conspicuous fluorescence was detected in association with the 

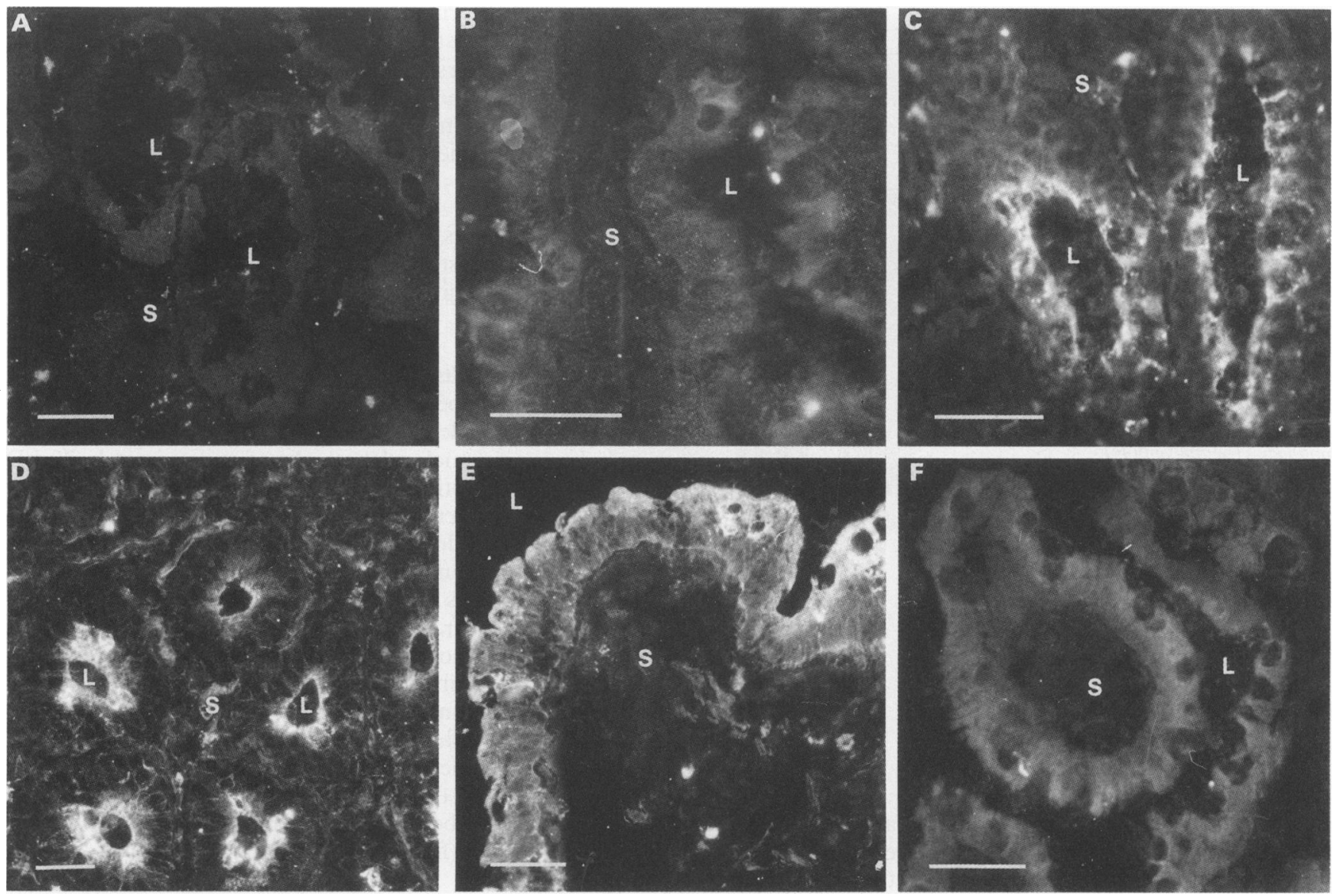

Figure 2: A pepsin trypsin digest of gliadin was instillated in the jejunum in healthy individuals and coeliac patients in remission. Intestinal biopsy specimens were obtained at different times and the uptake of the digest was investigated by immunofluorescence microscopy using affinity-purified antibodies. (A): coeliac patient before the instillation, villous region. $(B)$ : healthy individual after 20 minutes of instillation, villous region. $(C)$ : coeliac patient after 20 minutes of instillation, villous region. $(D)(E)$ : coeliac patient after 40 minutes of instillation, crypt region $(D)$ and villous region $(E)$. $(F)$ : coeliac patient 20 minutes after the instillation was terminated, villous region. The calibration bars indicate $25 \mu \mathrm{m}$. L: intestinal lumen; $S$ : submucosa.

lateral membrane/intercellular space of the enterocytes. The staining was most intense in the villous part, but weak colouring also occurred in the crypts. Intense staining was also seen intracellularly in the enterocytes (Fig 2C). This intracellular fluorescence was located at the brush border region and a granular staining was observed apically within the enterocytes (Fig 3). The applied immunofluorescence technique, however, did not allow a detailed description of the subcellular localisation of the internalised antigen.

The pattern changed after 40 minutes of instillation (Fig 2D, E). The subcellular location of the staining was similar but the staining in relation to the lateral membrane/intercellular space and the apical region of the cells was now more intense in the crypt region (Fig 2D) compared with the villous region (Fig $2 \mathrm{E}$ ). The last biopsy specimen in the series was taken after 60 minutes, and thus 20 minutes after the PTgliadin instillation was terminated. The staining, which was still most intense in the crypt region, was located in small granules not only near the microvillar membrane, but generally distributed all over the cytoplasm apically for the nucleus (Fig 2F). A marked staining at the lateral membranes could no longer be observed.

All sections were analysed with the three antibody dilutions. Noticeably, there was no apparent variation in staining intensity between the tested dilutions in the specimens from coeliac patients.

\section{Discussion}

The current study clearly shows for the first time that PT-gliadin and/or fragments hereof are present in normal enterocytes after exposure. The PT-gliadin is homogeneously distributed in the apical region of these cells. We have previously performed similar instillation experiments, using undigested gliadin (unpublished results). The gliadin was detected at the microvillar membrane, but an uptake could not be demonstrated. Accordingly, it seems as though only small fragments of gliadin can be taken up, and that an intraluminal digestion of orally ingested gliadin is a prerequisite for the uptake. Even though the intestinal brush border enzymes from healthy individuals in vitro are able to hydrolyse gliadin completely, ${ }^{6}$ our results show, that at least a proportion of the instillated PT-gliadin escapes complete hydrolysis in vivo. The demonstration of PT-gliadin in enterocytes of normal individuals shows that internalised PT-gliadin is not toxic per se.

An in vivo uptake of polypeptides by the human enterocytes has to our knowledge not been demonstrated previously, and the zonula occludens (tight junction) between the cells is usually considered impermeable to macro- 


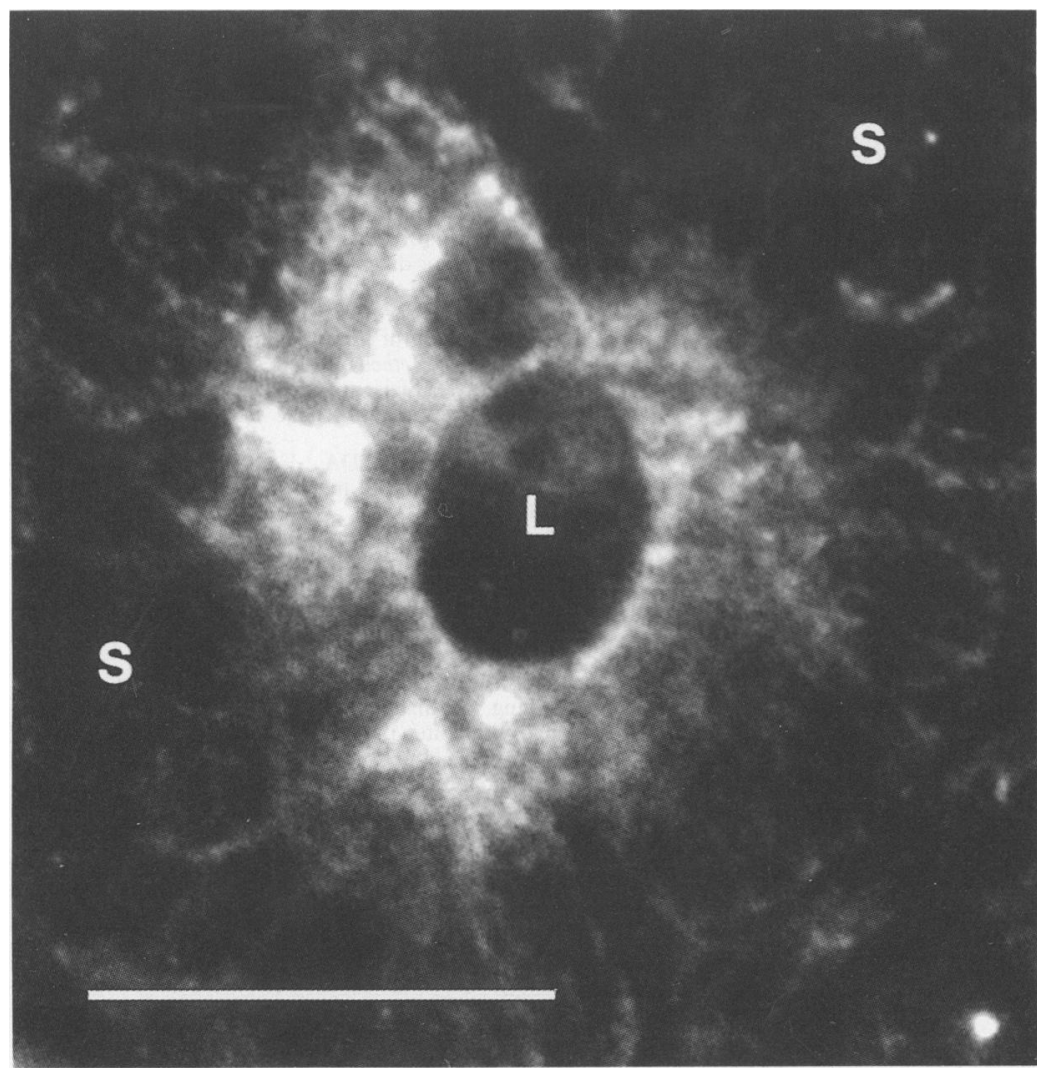

Figure 3: An intense intracellular staining was observed in the enterocytes from coeliac patient after 20 minutes of exposure to a pepsin trypsin digest of gliadin. The granular staining was only observed in the apical region of the cells. The calibration bar indicates $25 \mu \mathrm{m}$. L: intestinal lumen; S: submucosa. the tight junctions are functionally altered, thereby allowing PT-gliadin to pass into the intercellular space. The increased cellular uptake of PT-gliadin in coeliac patients could therefore occur indirectly through endocytosis at the lateral membrane. Accordingly, the results are in agreement with the observation that endocytosis from the basolateral membrane is more abundant in the crypt region than in the villous region ( $\mathrm{GH}$ Hansen, unpublished results). Experiments with instillation of undigested gliadin did not show intracellular gliadin or gliadin in relation with basolateral membrane/intercellular space (unpublished results). The explanation might be that a probable leak at the tight junction is relative, allowing only small molecules to pass into the intercellular space. An increased amount of intracellular antigen caused by a constitutional increased apical endocytosis and/or a disturbance in an intracellular degradation of PT-gliadin in coeliac patients, however, can not be excluded.

An increased permeability of the mucosa in patients with coeliac disease has previously been shown by Bjarnason et al. ${ }^{18}$ They showed that a majority of patients in remission have an increased absorption of ${ }^{51} \mathrm{Cr}$-labelled ethylenediaminetetra acetic acid (EDTA), suggesting that coeliac disease was caused by a primary defect in intestinal mucosal permeability. The authors suggested that the increased permeability may reflect alterations in the intercellular junctional complexes allowing enhanced entry into the intercellular and submucosal spaces. Our results support the hypothesis of a functional alteration in the zonula occludens in the intestinal epithelium of coeliac patients.

A less likely interpretation of the results demonstrating an increased and substantial amount of PT-gliadin intracellularly in granules in coeliac disease patients is compatible with a pathological binding of PT-gliadin to surface receptors and a disturbance in the further intracellular processing. The immunological events of the disease might subsequently be initiated after transcytosis of the receptor ligand complexes.

There are several possible fates for the endocytosed PT-gliadin also in coeliac disease patients. In a review Davidson and Bridges ${ }^{19}$ pointed out, that an enhanced uptake of gliadin peptides might initiate lysosomal damage, and/ or increased permeation into the mucosal and submucosal space might trigger a cascade of local immunological events. It has previously been shown that infiltration of lymphocytes in the submucosa occurs within only a few hours after gliadin challenge. ${ }^{2021}$ This argues for a local exposition of gliadin related antigens. The observations in the present study indicate a pathologically increased entry of PT-gliadin across the epithelium in coeliac disease allowing adverse immunological reactions to occur.

We thank Hanne L Hansen and Dorit Anthonsen for their skillful technical assistance. The project was part of a programme under the Biomembrane Research Center and was supported by gran from the Danish Cancer Society, the Lundbeck Foundation, the Danish Medical Council, the NOVO Foundation, the Ingrid Munkholm Foundation, the Jacob Madsens and Olga Madsens Foundation and the Alfred Benzon Foundation. et $a l^{16}$ showed that the tight junctions appeared anchanged in untreated coeliac tight junctions may correlate inversely with the permeability of the epithelium ${ }^{17}$ and it has been shown that the crypt tight junctions have fewer strands than the villous junctions. ${ }^{17}$ Therefore, the results of the current study may indicate that 
1 Frazer AC, Fletcher RF, Ross CAC, Shaw B, Sammons HG, Schneider R. Gluten-induced enteropathy: the effect of partially digested gluten. Lancet 1959; i: $252-5$.

2 Bramble MG, Zucoloto S, Wright NA, Record CO. Acute gluten challenge in treated adult coeliac disease: a morphometric and enzymatic study. Gut 1985; 26: 169-74.

3 Douglas AP, Booth CC. Digestion of gluten peptides by normal human jejunal mucosa and by mucosa from patients normal human jejunal mucosa and by mucosa from

4 Pittmann FE, Pollit RJ. Studies of jejunal mucosa digestion of peptic-tryptic digests of wheat protein in coeliac disease. Gut 1966; 7: 368-71.

5 Cornell $\mathrm{HJ}$, Rolles CJ. Further evidence of a primary mucosal defect in coeliac disease. Gut 1978; 19: 253-9.

6 Bruce G, Woodley JF, Swan CHJ. Breakdown of gliadin peptides by intestinal brush border from coeliac patients. Gut 1984; 25: 919-24.

7 Bronstein HD, Lorraine JH, Kowlessar OD. Enzymatic digestion of gliadin: the effect of the resultant peptides in celiac disease. Clin Chim Acta 1965; 14: 141-55.

8 Friis SU, Norén $O$, Sjöström H, Gudmand-Høyer E. Patients with coeliac disease have a characteristic gliadin antibody pattern. Clin Chim Acta 1986; 155: 133-41.

9 Bradford MM. A rapid and sensitive method for the quantitation of microgram quantities of protein utilizing the principle of protein-dye binding. Anal Biochem 1976; 72: principle

10 Friis SU. Enzyme-linked immunosorbent assay for quantitation of cereal proteins toxic in coeliac disease. Clin Chim Acta 1988; 178: 261-70.

11 Hjelm H, Hjelm K, Sjöquist T. Protein-A Staphylococcus Aureus. Its isolation by affinity chromatography and its use as an immunosorbent for isolation of immunoglobulins. FEBS Lett 1982; 28: 73-6.

12 Friis SU, Sjöström $\mathbf{H}$, Norén $\mathrm{O}$, Rüdiger $\mathrm{N}$, Anthonsen $\mathrm{D}$. The prolamin antibody reactivity against hordein polypep- tides in the sera from patients with coeliac disease. Clin Chim Acta 1988; 176: 241-50.

13 Shmerling DH. A questionnaire of the European Society for Paediatric Gastroenterology and Nutrition on coeliac disease. In: McNicholl B, McCarthy CF, Fottrell PF, eds. Perspectives in coeliac disease. Lancaster: MTP Press, 1978: 245-6.

14 Friis SU, Larsen $K$, Boserup J, Kristensen SD, Jarnum S, Rasmussen SN. Gliadin antibody titers in coeliac patients during short term provocations with bread made from wheat during short term provocations with bread made from wheat Hepatol 1990; 2: 361-5.

15 Johnson GD, Nogueria Araujo GMC. A simple method of reducing the fading of immunofluorescence during microscopy. F Immunol Methods 1981; 43: 349-50.

16 Rubin W, Ross L, Sleisenger MH, Weser E. An electron microscopic study of adult celiac disease. Lab Invest 1966; 15: $1720-47$

17 Claude P, Goodenough DA. Fracture faces of zonulae occludentes from 'tight' and 'leaky' epithelia. $f$ Cell Biol 1973; 58: 390-400

18 Bjarnason I, Peters TJ. A persistent defect in small intestinal permeability in coeliac disease demonstrated by a $51-\mathrm{Cr}$ permeability in coeliac disease demonstrated by a $51-0$

19 Davidson AGF, Bridges MA. Coeliac disease: a critical review of aetiology and pathogenesis. Clin Chim Acta 1987; 163: $1-40$

20 Anand BS, Piris J, Jerrome DW, Offord RE, Truelove CS. The timing of histological damage following a single challenge with gluten in treated coeliac disease. Quart $\mathcal{F}$ Med New Series $L$ 1981; 197: 83-94.

21 Leigh RL, Marsh MN, Crowe P, Kelly C, Gardner V, Gordon D. Studies of intestinal lymphoid tissue. IX. Dose dependent, gluten-induced lymphoid infiltration of coeliac jejunal epithelium. Scand f Gastroenterol 1985; 20: 715-9. 